\title{
Selective storage and maintenance of an object's features in visual working memory
}

\author{
GeOFFrey F. WoOdMan \\ Vanderbilt University, Nashville, Tennessee \\ AND \\ EDWARD K. VOGEL \\ University of Oregon, Eugene, Oregon
}

\begin{abstract}
It has been shown that we have a highly capacity-limited representational space with which to store objects in visual working memory. However, most objects are composed of multiple feature attributes, and it is unknown whether observers can voluntarily store a single attribute of an object without necessarily storing all of its remaining features. In this study, we used a masking paradigm to measure the efficiency of encoding, and neurophysiological recordings to directly measure visual working memory maintenance while subjects viewed multifeature objects and were required to remember only a single feature or all of the features of the objects. We found that measures of both encoding and maintenance varied systematically as a function of which object features were task relevant. These experiments show that individuals can control which features of an object are selectively stored in working memory.
\end{abstract}

Our ability to temporarily maintain information is limited by the small capacity of approximately three to four objects of visual working memory (VWM; Irwin \& Andrews, 1996; Luck \& Vogel, 1997; Vogel, Woodman, $\&$ Luck, 2001). This seemingly unworkable limitation can be overcome by powerful selection mechanisms that regulate access to VWM so that only the most relevant objects consume its capacity (Chun \& Potter, 1995; Potter, 1976; Schmidt, Vogel, Woodman, \& Luck, 2002; Vogel, Luck, \& Shapiro, 1998). Although individuals differ in how effectively they implement these processes, it is clear that we can control which objects are held in VWM on the basis of current task goals (Vogel, McCollough, \& Machizawa, 2005). However, most objects in the environment are composed of multiple features, and it is unclear whether we obligatorily encode all of an item's features into VWM when an object is selected or whether only the task-relevant features are stored in memory. That is, when asked to remember only the color of an object, must we also represent all of the other attributes of the object, such as its shape and texture?

Evidence from the object-based attention literature appears to support the notion of obligatory storage of all features of an attended object. These studies often report that attention to an object automatically activates an object representation containing all of its features (Duncan, 1984; O’Craven, Downing, \& Kanwisher, 1999; Vecera \& Farah, 1994). Indeed, previous work examining VWM capacity could be interpreted as supporting this general view. Spe- cifically, we presented arrays of multifeatured objects (e.g., color, orientation) and asked subjects to remember either one feature or all of the features of objects (Luck \& Vogel, 1997; Vogel et al., 2001). We found that subjects were just as accurate at remembering all object features as they were at remembering single features. We took this as evidence that the storage capacity of VWM is determined by the number of objects in memory rather than the amount of feature information represented. However, this conclusion rests on one critical, but untested assumption: that in the single-feature conditions, subjects were capable of remembering just the one feature from each object without necessarily storing the other visible, but irrelevant features. This seems plausible, given that previous studies of temporary memory storage (Stefurak \& Boynton, 1986) and betweentrial priming during visual search (e.g., Kristjansson, 2006a; Maljkovic \& Nakayama, 1994) suggest that such feature-selective processing can occur. Consequently, an alternative interpretation of these results is that subjects are incapable of selectively remembering only one feature of an object and thereby obligatorily store all features, which would explain why there was equivalent performance in the single-feature and multifeature conditions.

In contrast to the obligatory storage hypothesis, there is considerable neurophysiological evidence that cortical neurons preferentially represent only the task-relevant properties of objects during memory retention intervals (see, e.g., Rainer, Asaad, \& Miller, 1998; Rao, Rainer, \& Miller, 1997; Sereno \& Amador, 2006). Although these

G. F.Woodman, geoffrey.f.woodman@vanderbilt.edu 
studies generally do not claim that these effects are specifically due to representation in VWM, these results suggest that VWM can flexibly encode and actively maintain only the task-relevant features of an object. Excluding task-irrelevant features of objects from VWM may result in significant advantages for information processing. Specifically, this more economic storage system may allow for faster encoding rates because not all object features always need to be encoded, or it may afford faster changedetection responses by decreasing the response competition from irrelevant-feature information in memory.

In the present study, we attempt to distinguish between these alternative accounts by using two new techniques, each targeting a different aspect of VWM processingnamely, VWM encoding (also known as consolidation) and VWM maintenance. By examining both processes, we can more completely characterize the fate of the irrelevant object features.

To examine VWM encoding, we utilized a psychophysical technique in which pattern masks limit the period the observer has to encode information on each trial. This work builds on classic studies of iconic and short-term memory storage that used attentional cues and focused on shorter target-to-mask SOAs (Averbach \& Coriel, 1961; Gegenfurtner \& Sperling, 1993; Sperling, 1960). We varied the stimulus onset asynchrony (SOA) between the memory and masking arrays to estimate the time required to form a durable representation in VWM capable of persisting despite new object onsets, unlike iconic memory representations (Phillips, 1974). This approach has provided novel insights into the time course of VWM encoding across different stimulus attributes. Vogel, Woodman, and Luck (2006) found that the encoding rate for colored squares was approximately $50 \mathrm{msec} / \mathrm{object}$. In contrast, Woodman and Vogel (2005) found that the encoding rate for orientations and shapes was much slower, approximately $80-100 \mathrm{msec} / \mathrm{object}$. Although we are cautious about making fine comparisons across experiments, these disparate encoding rate estimates may provide a means for testing whether task-irrelevant features are encoded into VWM. Specifically, we measured the VWM encoding rate for colored, oriented rectangles and instructed observers to remember only the color, only the orientation, or both features of each object. If all object features are obligatorily encoded, then we expect all three conditions to produce equivalent encoding rates. However, if observers can flexibly encode only the task-relevant features of an object in VWM, then the encoding rates for color and orientation conditions should differ, in line with our previous estimates. This would indicate that despite the presence of task-irrelevant features, the encoding rate is determined strictly by the task-relevant object features.

We further tested these hypotheses using a new neurophysiological technique for directly measuring the information that is actively maintained in VWM. This measure is a sustained, lateralized event-related potential (ERP) component known as the contralateral delay activity (CDA) because of its similarity to delay activity exhibited by cortical neurons during delayed-match-to-sample tasks (Bisley \& Goldberg, 2003; Chaffee \& Goldman-Rakic,
1998; Miller \& Desimone, 1991; Miller, Erickson, \& Desimone, 1996; Miller, Li, \& Desimone, 1993). The CDA is a large negative wave maximal at posterior-parietal electrodes over the hemisphere contralateral to the position of remembered items. This activity is sustained throughout the memory retention interval of change-detection tasks and appears to reflect the representations held in VWM. The amplitude of the CDA increases with the number of items held in memory, with the asymptote being each individual's VWM capacity limit (Vogel \& Machizawa, 2004). Moreover, the overall amplitude (regardless of array size) of the CDA varies across different stimulus attributes, with larger amplitudes observed for orientation information than for color (McCollough, Machizawa, \& Vogel, 2007). Thus, CDA amplitude can provide a sensitive measure of both the quantity and the type of information maintained in VWM. We measured CDA amplitude while observers remembered only the color, only the orientation, or both the color and orientation of each object, to determine whether the VWM representations are the same across conditions or whether only the task-relevant object attributes are held in memory.

\section{METHOD}

\section{Psychophysical Methods}

Twenty and 32 Vanderbilt undergraduates participated in the first and second masking experiments, respectively. All were 18-32 years old, provided informed consent, and had normal or corrected-tonormal visual acuity.

The stimuli were viewed on a gray background $\left(40.6 \mathrm{~cd} / \mathrm{m}^{2}\right)$ at a distance of $\sim 57 \mathrm{~cm}$. In the first masking experiment, the sample arrays consisted of three colored, oriented bars $\sim 2.0^{\circ} \times 0.4^{\circ}$ of visual angle. Each object was centered $7.2^{\circ}$ from fixation, randomly placed at one of 12 clock-face-like possible locations (see Figure 1A). The orientation and color of each bar was randomly selected (without replacement) from the set of four orientations (vertical, horizontal, tilted $-45^{\circ}$, or tilted $+45^{\circ}$ ) and seven possible colors (using CIE 1931 color space; red, $x=.627, y=.327$; blue, $x=.142, y=.065$; violet, $x=.279, y=.139$; green, $x=.280, y=.589$; yellow, $x=$ $.397, y=.500$; black, $0.04 \mathrm{~cd} / \mathrm{m}^{2}$; and white, $92.6 \mathrm{~cd} / \mathrm{m}^{2}$ ). The mask array was composed of three checkerboard-like masks generated by randomly selecting (with two replacements) a color for each cell (approximately $\left.0.6^{\circ} \times 0.6^{\circ}\right)$ of a $4 \times 4$ matrix. One mask was centered on each sample stimulus location.

On each trial, a fixation point $\left(0.04 \mathrm{~cd} / \mathrm{m}^{2}, 0.05^{\circ} \times 0.05^{\circ}\right)$ appeared $500 \mathrm{msec}$ before the sample array. The sample array was presented for $\sim 23 \mathrm{msec}$ and its offset was followed $35,105,140$, or $176 \mathrm{msec}$ later by the onset of the mask array. The mask array was shown for $500 \mathrm{msec}$ during the retention interval and the screen was blank for a variable duration following mask array offset such that the retention interval was always $1,500 \mathrm{msec}$. The test array was extinguished by the observer's response or after 5,000 msec.

In the color, orientation, and conjunction conditions, the sample stimuli were identical and only the instructions of which features were relevant and the types of possible changes differed. In each condition, subjects performed 16 practice trials followed by three 48-trial experimental blocks. Accuracy was stressed and condition order was randomized across participants. Change-detection performance was measured using the $K$ metric (Pashler, 1988), derived from hit and false alarm rate (Cowan, 2001; Woodman \& Vogel, 2005 ), for estimating the number of objects represented in memory.

The methods of the second masking experiment were identical to the first except for the use of seven shape stimuli (see Figure 1B; see also Woodman \& Vogel, 2005) and the inclusion of more observers 


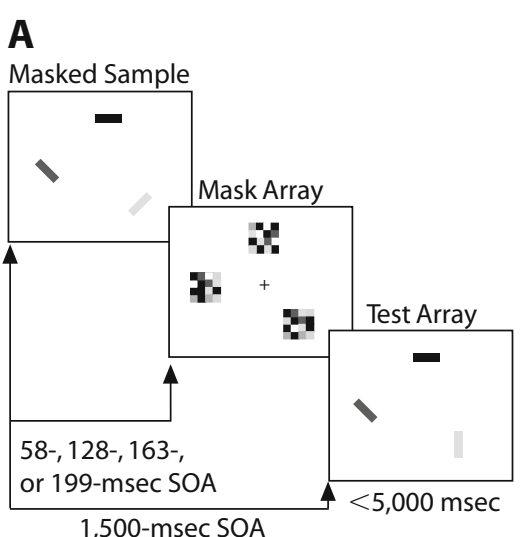

B

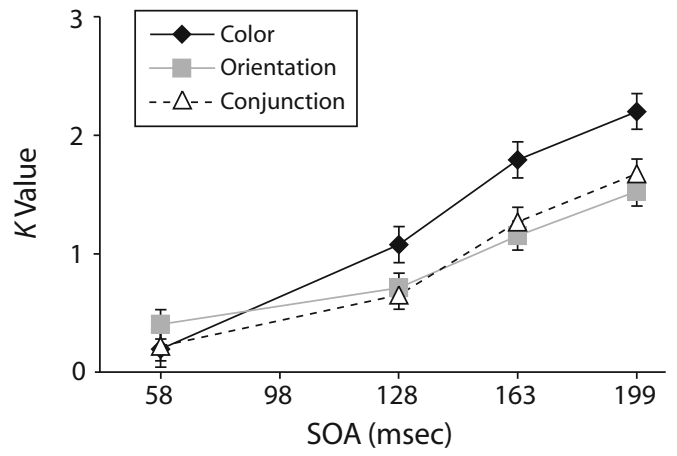

C

Masked Sample

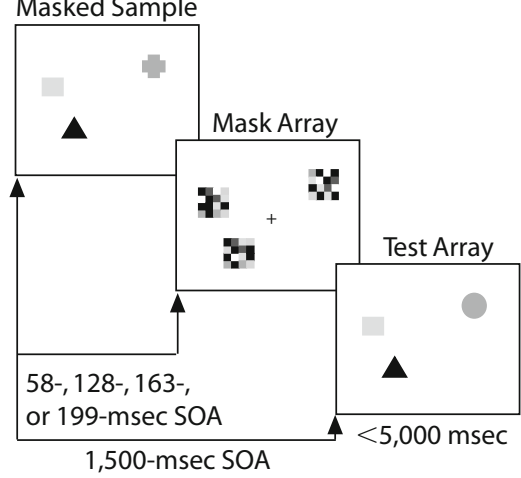

D

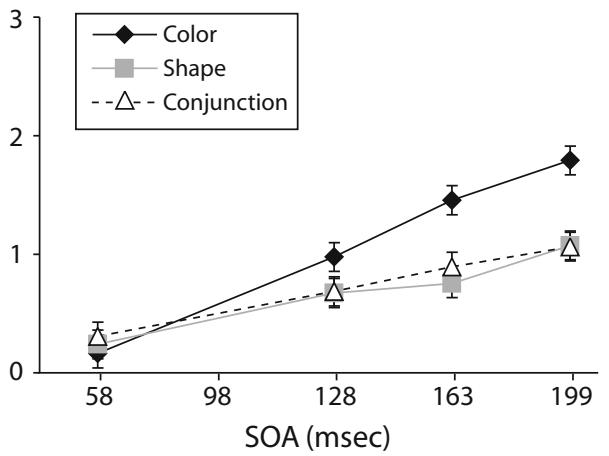

Figure 1. Examples of the stimuli and the results from the psychophysical masking experiments. (A) Example of the masked change-detection paradigm used in the first experiment. The test arrays were terminated by the subject's response or were extinguished after $5 \mathrm{sec}$. (B) Data from the first experiment, showing the mean $K$ value across observers for each condition and SOA. (C) Example of the stimulus sequence of the second masking experiment. For the example trials in $A$ and $C$, the correct response is "different," due to orientation and shape changes, respectively. (D) The $K$ values' measurements from the second psychophysical experiment. Error bars represent the $95 \%$ within-subjects confidence intervals (as recommended in Loftus \& Loftus, 1988).

$(N=32)$. In both experiments, observers simultaneously performed articulatory suppression to prevent verbal recoding (see Woodman \& Vogel, 2005).

\section{Electrophysiological Methods}

Ten University of Oregon undergraduates (ages 19-26 years) participated in the ERP experiment after giving informed consent. Each observer performed 220 trials per condition. The factor of array size (two vs. four items) was mixed within blocks, whereas task condition (color, orientation, and conjunction) varied between blocks and the condition order was randomized.

Beginning each trial, a central arrow cue pointed left or right, instructing the observer to remember the items in one hemifield. After $250 \mathrm{msec}$, the memory array, composed of either two or four oriented rectangles in each hemifield, was presented for $100 \mathrm{msec}$. Memory array items were presented within two $4^{\circ} \times 7.3^{\circ}$ regions centered $3^{\circ}$ to the left and right of a central fixation cross on a gray background $\left(8.2 \mathrm{~cd} / \mathrm{m}^{2}\right)$. Each rectangle $\left(0.65^{\circ} \times 2^{\circ}\right)$ was randomly selected (with one replacement) from a set of four orientations (vertical, horizontal, left $45^{\circ}$, and right $45^{\circ}$ ) and four colors (red, green, blue, and black). Stimulus positions were randomized, with at least $2^{\circ}$ between objects. After a 1,000-msec retention interval, the test array was presented for $2,000 \mathrm{msec}$. On half of the trials, the test array was identical to the memory array, and on the remaining trials, it differed by one feature of one item.
ERPs were recorded and analyzed using standard procedures (McCollough et al., 2007; Vogel et al., 1998). Trials contaminated by blinks or $>1^{\circ}$ eye movements (mean $=15 \%$ of trials per subject) were rejected. We recorded from 22 standard electrode sites. ERPs were time-locked to memory array onset and recorded throughout the retention period until test array presentation. The CDA was measured as the difference in mean amplitude between the ipsilateral and contralateral waveforms (PO1/PO2), from 300 to $900 \mathrm{msec}$ following memory array onset.

\section{RESULTS}

\section{Feature-Selective Consolidation}

In our first psychophysical experiment using colored, oriented rectangles (Figure 1A), change-detection performance rose more quickly as the sample-to-mask SOA increased in the color condition than it did in the orientation condition (Figure 1B). Moreover, the consolidation rate in the conjunction condition was similar to that of the orientation condition. The two-way ANOVA with the factors of condition (color, orientation, or conjunction) and SOA $(35,107,140$, or $175 \mathrm{msec})$ yielded significant main effects of condition $[F(2,38)=9.93, p<.001]$ and 
SOA $[F(3,57)=94.92, p<.0001]$ and an interaction of these factors $[F(6,114)=5.02, p<.001]$. Planned comparisons between conditions supported the observations that the color condition differed from both the orientation and conjunction conditions $[F(1,19)=10.90, p<.01$, and $F(1,14)=23.20, p<.001$, respectively], and both interactions with SOA were significant $[F(3,57)=8.41$, $p<.001$, and $F(3,57)=4.39, p<.01$, respectively]. The orientation and conjunction conditions did not differ from each other, nor was there an interaction with SOA ( $p$ s $>$ $.25)$. In all comparisons, the effect of SOA was highly significant $(p s<.0001)$.

We also evaluated the selectivity of VWM encoding by computing the slope of the consolidation functions in terms of the amount of time needed to consolidate each item. The slope of the color consolidation function was $67.7 \mathrm{msec} /$ item, and differed from the consolidation slopes in the other two conditions $(p \mathrm{~s}<.05)$. The slopes in the orientation (117.7 msec/item) and conjunction ( $96.3 \mathrm{msec} /$ item $)$ conditions were not significantly different $(p>.30)$.

This first experiment shows that the consolidation rate in the color condition was faster than in the orientation condition, despite identical stimuli across conditions. These findings support the flexible-encoding hypothesis that VWM consolidation can be limited to just the to-beremembered features of objects, and are contrary to the predictions of a rigid-consolidation process. Consolidation rate in the conjunction condition was no slower than the rate for the slower-to-encode component feature. These findings suggest that VWM encoding is best accounted for by a mechanism that can process multiple features in parallel, but with limited capacity.

In our second psychophysical experiment, a new, larger group of observers detected changes in the color, geometric shape, or both the color and shape of a set of items (Figure 1C) to replicate the first experiment and allow for analysis of the different types of feature changes in the conjunction condition. Identical to the results of our first experiment, change-detection performance rose more rapidly across SOA in the color condition than in the shape or conjunction conditions (Figure 1D), resulting in a significant effect of condition $[F(2,62)=22.68, p<.001]$ and SOA $[F(3,93)=95.27, p<.001]$ and a significant interaction of these factors $[F(6,186)=8.95, p<.001]$. Planned comparisons confirmed that the color condition differed from the shape $[F(1,31)=33.89, p<.0001]$ and conjunction $[F(1,31)=28.34, p<.0001]$ conditions. However, the shape and conjunction conditions did not significantly differ from one another $(F<1)$. The effect of SOA was significant in all pairwise tests of conditions $(p \mathrm{~s}<.0001)$. The slope of the color consolidation function was $83.6 \mathrm{msec} /$ item and differed significantly from the shape slope $(170.2 \mathrm{msec} / \mathrm{item}, p<.05)$ and the conjunction condition slope (181.6 msec/item, $p<.01$ ), but the shape and conjunction conditions did not differ $(F<1)$. Thus, identical patterns of effects were observed in both psychophysical masking experiments.

By focusing on only the change trials, we can test the hypothesis that consolidation of color is slowed when shape must also be encoded, as conjunction performance sug- gests. Figure 2 shows the percentage of changes detected in the conjunction condition and feature conditions. The slope of the function relating change-detection accuracy, in percentage correct, to SOA for color changes in the color condition was significantly steeper than for color changes detected in the conjunction condition $(p<.05)$. In contrast, the slopes of the shape-change consolidation functions did not differ significantly between the shape and conjunction conditions $(F<1)$. These findings show that the consolidation of color was slowed when shape also had to be encoded, and further support the proposal that consolidation efficiency is dependent on whether other visual features of the objects are task relevant.

\section{Feature-Selective Storage}

In our last experiment, we used an alternative approach to test the flexible versus obligatory storage hypotheses. With psychophysical methods alone, it is possible that results are due to processing at late stages, such as response selection, meaning that the VWM representations stored were actually identical between conditions in the first experiments. To address this possible limitation, we used the CDA component as an electrophysiological measure of what was maintained in VWM during the retention intervals. The stimuli were presented within a bilateral display, and observers were asked to remember the color and orientation of both features, across different blocks of trials, of the items within the cued hemifield (see Figure 3A).

Figure 3B shows the behavioral performance in this task. Replicating previous reports, observers were equally good at detecting changes in color, orientation, and conjunction conditions $(F<1)$, and overall performance decreased with the set size increase from two to four items $(p<.01)$ (Luck \& Vogel, 1997; Vogel et al., 2001).

To isolate the CDA, we time-locked the ERPs to memory array onset and constructed difference waves by subtracting ipsilateral activity from activity contralateral to the cued hemifield. Figure 3C shows these difference waveforms across the task conditions. As shown previ-

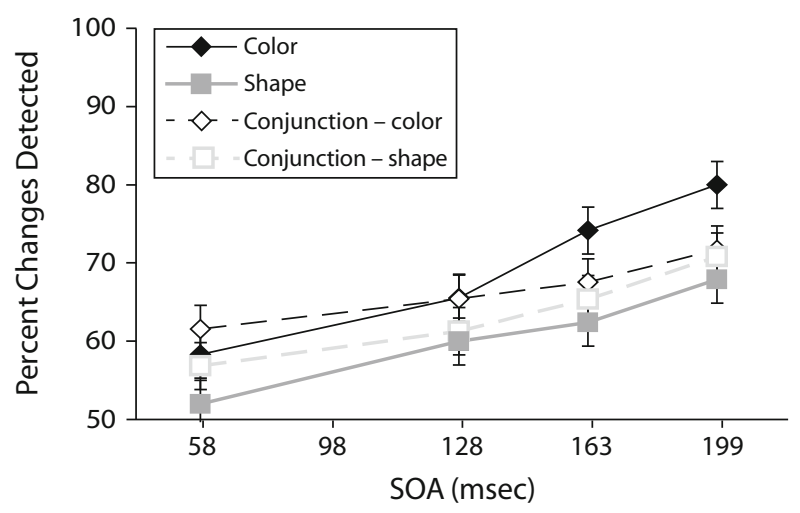

Figure 2. Accuracy with which changes were detected (percentage of changes detected) in the feature and conjunction conditions of the second psychophysical experiment. Diamonds represent color changes, squares represent shape changes, filled shapes show changes in the feature conditions, and unfilled shapes show changes in the conjunction condition. 


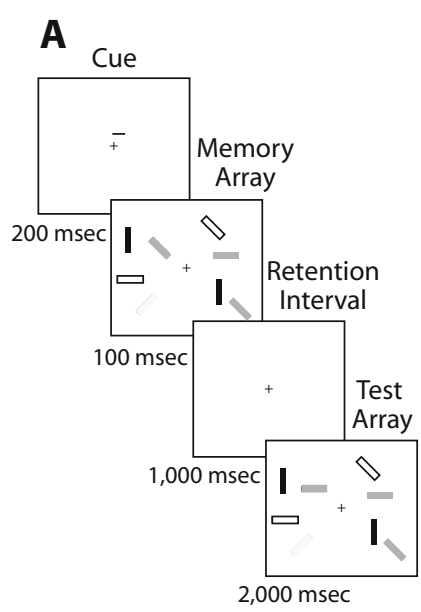

\section{B}
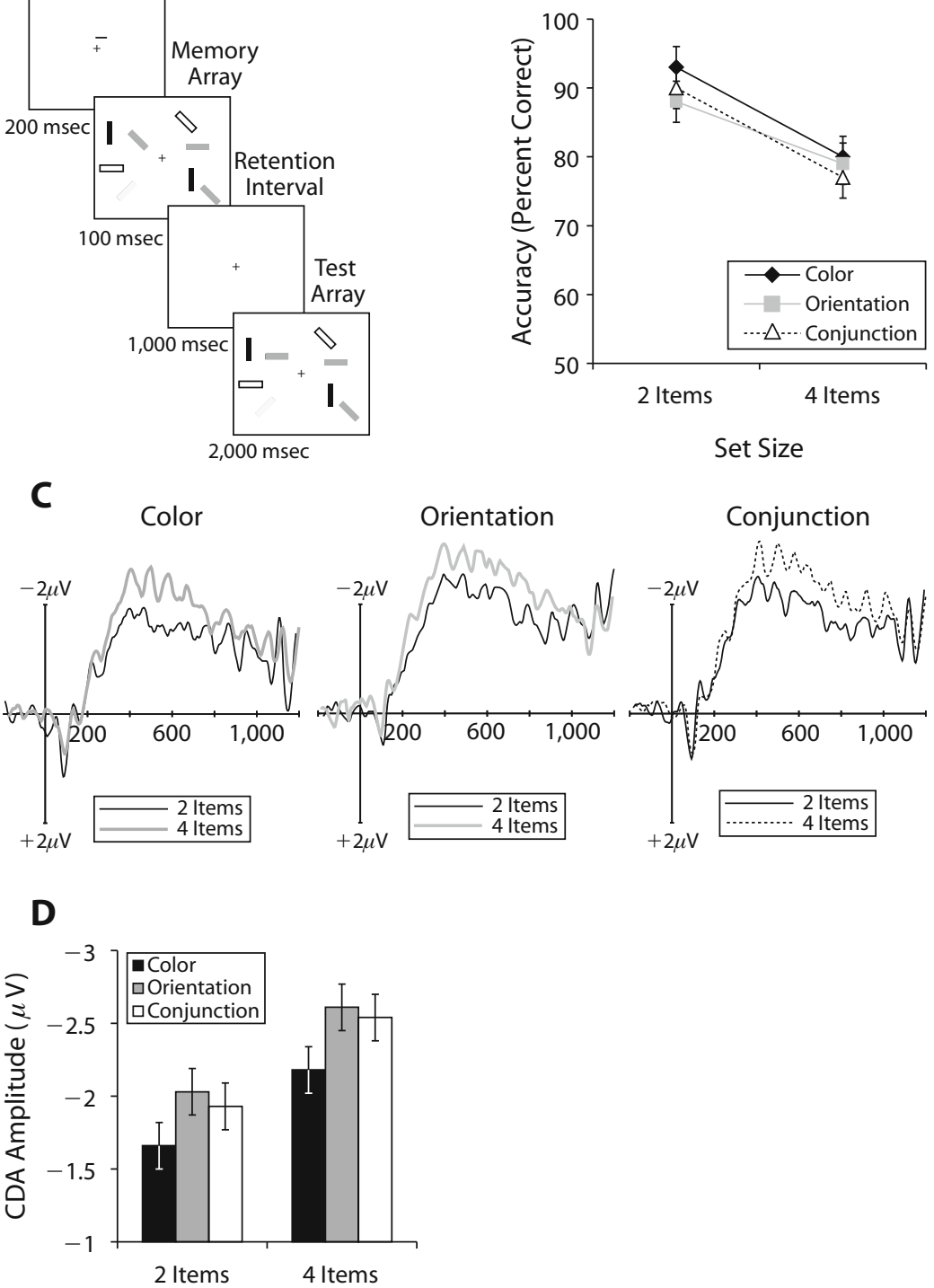

Figure 3. Stimuli and results from the electrophysiological experiment. (A) An example of the stimulus sequence presented while ERPs were recorded. (B) Accuracy of change-detection performance in percentage correct as a function of the number of items to be remembered. (C) The ipsilateral-contralateral ERP waveforms from the color, orientation, and conjunction conditions showing the CDA component when two or four items were to be remembered. The waveforms were recorded from electrode sites PO1/PO2 in the standard 10/20 system configuration. For presentation only, the ERP waveforms were digitally low-pass filtered by convolving the waveforms with a Gaussian impulse response function $(S D=6 \mathrm{msec}, 50 \%$ amplitude cutoff at $30 \mathrm{~Hz}$ ). (D) Bar graph of the CDA amplitude measured from 200 to 800 msec after the memory stimulus array offset.

ously, the CDA amplitude was larger during memory retention of four-item as opposed to two-item arrays. Importantly, the overall amplitude (regardless of array size) was larger for the orientation and conjunction conditions than for the color condition. An ANOVA on mean CDA amplitude from 300 to $900 \mathrm{msec}$ post-memory array onset (Figure 3D) supported these observations and yielded significant main effects of array size $[F(1,12)=11.25, p<.01]$ and condition $[F(2,24)=6.40, p<.01]$ but no interaction between these factors $(F<1)$. Planned comparisons revealed significant differences between the color condition and the orientation and conjunction conditions $(p \mathrm{~s}<.01)$, but no difference between the orientation and conjunction conditions $(p>.15)$.

Although subjects viewed identical stimuli, CDA amplitude was differentially modulated depending on 
whether the color or orientation was task relevant. If subjects obligatorily store all features of an object in VWM, one would expect no amplitude difference across the task conditions. However, the larger amplitude CDA in the orientation than in the color condition indicates that subjects were indeed able to selectively store only the color information from the objects, despite the presence of orientation information. These results strongly favor the selective feature storage hypothesis.

\section{Individual Differences in Feature Selection?}

Previous research has shown that individual differences in VWM capacity predict subjects' ability to control which objects are retained in VWM (Vogel et al., 2005). Here, we tested whether the ability to select between relevant and irrelevant features of objects might also vary with VWM capacity. Thus, we performed a median split of the subjects based on behavioral performance at the longest masking SOA (199 msec) or largest ERP set size (4). In the psychophysical experiments, the low-capacity observers were generally slower than the high-capacity subjects at consolidating information into VWM, but both groups encoded color more efficiently than orientation/shape or the conjunction condition, with the form feature and conjunction encoding efficiency being equivalent. Both ANOVAs with the between-subjects variable of high versus low capacity yielded no significant interactions of capacity $X$ condition $\times$ SOA for either of the psychophysical masking experiments $\left(F_{\mathbf{S}}<1\right)$, despite significant main effects of all of these factors $(p s<.01)$.

The electrophysiological data showed the same pattern. The CDA amplitude differences between remembering color versus orientation or the conjunction of features did not differ between high- and low-capacity subjects. The ANOVA yielded no significant capacity $\times$ condition interaction $(F<1)$, despite a significant capacity $\times$ set size interaction $(p<.01)$, with high-capacity subjects showing larger amplitude differences between set size 2 and set size 4 than did low-capacity subjects, replicating previous work (Vogel \& Machizawa, 2004). Although further studies with larger samples will be necessary to confirm these observations, the present findings suggest that whereas VWM capacity differences correlate with the ability to control which objects are stored in VWM, the ability to selectively encode specific feature information within an object is common across subjects and is not correlated with an individual's memory capacity.

\section{Advantages of Feature-Selective Storage?}

It is reasonable to wonder why VWM would utilize selective storage of object features, given that more feature information could be stored without incurring additional storage capacity limits. Our findings suggest that selective storage has at least two advantages over a system in which all object features are obligatorily encoded and stored. First, when only a subset of features needs to be remembered, this information can be more quickly encoded, or consolidated, into a robust form in VWM. The results of the masking experiments provide a demonstration of this. Second, a selective storage VWM system could prevent interference from irrelevant information at the response stage of processing. As more features of each object are stored, additional decisions might be necessary, increasing the probability of an error or slowing response selection. The reaction time data collected during the ERP experiment suggest that this may be the case. Observers were fastest to correctly respond to the test array in the singlefeature conditions (color RT, set size $2=664 \mathrm{msec}$, set size $4=773$; orientation RT, set size $2=706 \mathrm{msec}$, set size $4=805 \mathrm{msec}$ ) as opposed to the conjunction condition (set size $2=725 \mathrm{msec}$, set size $4=830 \mathrm{msec}$ ), resulting in significant RT effects of condition and set size $(p s<.05)$. These findings suggest that selective storage in VWM affords faster processing both during encoding and during response-related operations.

\section{DISCUSSION}

We contrasted the hypothesis that VWM mechanisms select which object features are stored on the basis of the current task with the obligatory storage hypothesis, in which storing any feature of an object requires storage of all of the object's features. The findings of the masking and electrophysiological experiments converge to support the selective feature storage hypothesis. Three aspects of these findings are most relevant for models of VWM.

First, the rate of consolidation varied as a function of which feature was task relevant. Despite identical physical stimuli between conditions, the color-memory tasks resulted in steeper consolidation slopes than did the shape- or orientation-memory tasks. These findings indicate that consolidation is selective for the task-relevant features and, therefore, under top-down control. Our ERP experiment supports this conclusion in showing that after encoding, only the task-relevant features are maintained in VWM (see also Kristjansson, 2006b).

Second, these findings speak to whether the consolidation of features takes place serially or in parallel. When observers were required to remember feature conjunctions, the consolidation rate was essentially identical to the consolidation rate of the slower-to-encode feature. If the consolidation of separate features required serial processing, then consolidating multiple features should have had an additive effect in slowing the consolidation rate relative to the slowest single-feature condition; however, this was not obtained.

Third, the present findings are relevant for the debate over the nature of the representations in VWM. The observation that the consolidation of conjunctions progresses at the same rate as the consolidation of the slower-to-encode features is particularly relevant. This could be accounted for in at least two ways. First, this could be due to VWM consolidation mechanisms that operate on bound-object representations when all features are task relevant (e.g., Irwin \& Andrews, 1996; Vogel et al., 2001), so that representations can only be encoded into VWM as quickly as the slowest-to-encode feature. Feature-based models of VWM would account for these data by proposing separate consolidation mechanisms that simultaneously fill independent-feature stores (e.g., Wheeler \& Treisman, 
2002). However, their simultaneous operation causes color encoding to be slowed in the conjunction condition. Thus, the present findings constrain these competing models of VWM representation.

\section{AUTHOR NOTE}

This research was supported by National Research Service Award F32-EY015043 and a Vanderbilt University Discovery Grant to G.F.W. and NSF Grant BCS-0617681 to E.K.V. We thank Jay Pratt, Molly Potter, Rhodri Cusack, and an anonymous reviewer for helpful comments and suggestions. Correspondence concerning this article should be addressed to G. F. Woodman, Department of Psychology, Wilson Hall, 111 21st Avenue South, Vanderbilt University, Nashville, TN 37240-1103 (e-mail: geoffrey.f.woodman@vanderbilt.edu).

\section{REFERENCES}

Averbach, E., \& Coriel, A. S. (1961). Short-term memory in vision. Bell System Technical Journal, 40, 309-328.

BisLey, J. W., \& GoldBerg, M. E. (2003). Neuronal activity in the lateral intraparietal area and spatial attention. Science, 299, 81-86.

Chaffee, M. V., \& Goldman-Rakic, P. S. (1998). Matching patterns of activity in primate prefrontal area $8 \mathrm{a}$ and parietal area 7ip neurons during a spatial working memory task. Journal of Neurophysiology, 79, 2919-2940.

Chun, M. M., \& PotTer, M. C. (1995). A two-stage model for multiple target detection in rapid serial visual presentation. Journal of Experimental Psychology: Human Perception \& Performance, 21, 109-127.

Cowan, N. (2001). The magical number 4 in short-term memory: A reconsideration of mental storage capacity. Behavioral \& Brain Sciences, 24, 87-185.

DunCAN, J. (1984). Selective attention and the organization of visual information. Journal of Experimental Psychology: General, 113, 501-517.

Gegenfurtner, K. R., \& Sperling, G. (1993). Information transfer in iconic memory experiments. Journal of Experimental Psychology: Human Perception \& Performance, 19, 845-866.

IRWIN, D. E., \& ANDREWs, R. V. (1996). Integration and accumulation of information across saccadic eye movements. In T. Inui \& J. L. McClelland (Eds.), Attention and performance XVI: Information integration in perception and communication (pp. 125-155). Cambridge, MA: MIT Press, Bradford Books.

KRISTJANSSON, A. (2006a). Simultaneous priming along multiple dimensions in a visual search task. Vision Research, 46, 2554-2570.

KRISTJANSSON, A. (2006b). Surface assignment modulates object formation for visual short-term memory. Perception, 35, 865-881.

Loftus, G. R., \& Loftus, E. F. (1988). Essence of statistics (2nd ed.). New York: Random House.

LuCK, S. J., \& VogeL, E. K. (1997). The capacity of visual working memory for features and conjunctions. Nature, 390, 279-281.

Maljkovic, V., \& Nakayama, K. (1994). Priming of pop-out: I. Role of features. Memory \& Cognition, 22, 657-672.

McCollough, A. W., Machizawa, M. G., \& Vogel, E. K. (2007). Electrophysiological measures of maintaining representations in visual working memory. Cerebral Cortex, 43, 77-94.

Miller, E. K., \& Desimone, R. (1991). A neural mechanism for working and recognition memory in inferior temporal cortex. Science, 254, 1377-1379.

Miller, E. K., Erickson, C. A., \& Desimone, R. (1996). Neural mecha- nisms of visual working memory in prefrontal cortex of the macaque. Journal of Neuroscience, 16, 5154-5167.

Miller, E. K., Li, L., \& Desimone, R. (1993). Activity of neurons in anterior inferior temporal cortex during a short-term memory task. Journal of Neuroscience, 13, 1460-1478.

O'Craven, K. M., Downing, P. E., \& Kanwisher, N. (1999). fMRI evidence for objects as the units of attentional selection. Nature, 401, 584-587.

Pashler, H. (1988). Familiarity and visual change detection. Perception \& Psychophysics, 44, 369-378.

Phillips, W. A. (1974). On the distinction between sensory storage and short-term visual memory. Perception \& Psychophysics, 16, 283-290.

Potter, M. C. (1976). Short-term conceptual memory for pictures. Journal of Experimental Psychology: Human Learning \& Memory, 2, 509-522.

Rainer, G., AsaAd, W. F., \& Miller, E. K. (1998). Selective representation of relevant information by neurons in the primate prefrontal cortex. Nature, 393, 577-579.

RAO, S. C., RAINER, G., \& MilLER, E. K. (1997). Integration of what and where in the primate prefrontal cortex. Science, 276, 821-824.

Schmidt, B. K., Vogel, E. K., Woodman, G. F., \& Luck, S. J. (2002). Voluntary and automatic attentional control of visual working memory. Perception \& Psychophysics, 64, 754-763.

SERENO, A. B., \& Amador, S. C. (2006). Attention and memory-related responses of neurons in the lateral intraparietal area during spatial and shape-delayed match-to-sample tasks. Journal of Neurophysiology, 95, 1078-1098.

SPERLING, G. (1960). The information available in brief visual presentations. Psychological Monographs, 74(Whole No. 498).

Stefurak, D. L., \& Boynton, R. M. (1986). Independence of memory for categorically different colors and shapes. Perception \& Psychophysics, 39, 164-174.

Vecera, S. P., \& Farah, M. J. (1994). Does visual attention select objects or locations? Journal of Experimental Psychology: General, 123, 146-160.

Vogel, E. K., LuCK, S. J., \& ShaPIRo, K. L. (1998). Electrophysiological evidence for a postperceptual locus of suppression during the attentional blink. Journal of Experimental Psychology: Human Perception \& Performance, 24, 1656-1674.

Vogel, E. K., \& Machizawa, M. G. (2004). Neural activity predicts individual differences in visual working memory capacity. Nature, 428, 748-751.

Vogel, E. K., McCollough, A. W., \& Machizawa, M. G. (2005). Neural measures reveal individual differences in controlling access to working memory. Nature, 438, 500-503.

Vogel, E. K., Woodman, G. F., \& LuCK, S. J. (2001). Storage of features, conjunctions, and objects in visual working memory. Journal of Experimental Psychology: Human Perception \& Performance, 27, 92-114.

Vogel, E. K., Woodman, G. F., \& LucK, S. J. (2006). The time course of consolidation in visual working memory. Journal of Experimental Psychology: Human Perception \& Performance, 32, 1436-1451.

WheEler, M., \& Treisman, A. M. (2002). Binding in short-term visual memory. Journal of Experimental Psychology: General, 131, 48-64.

Woodman, G. F., \& Vogel, E. K. (2005). Fractionating working memory: Encoding and maintenance are independent processes. Psychological Science, 16, 106-113.

(Manuscript received March 19, 2007; revision accepted for publication June 25, 2007.) 